\title{
NeuroRehab
}

News

\section{Realidad virtual \\ en el abordaje de alteraciones vestibulares}

Realidad Virtual

\section{Resumen:}

as alteraciones vestibulares parece que se encuentran relacionadas con el envejecimiento de la L población. En el intento de poder mejorar la calidad de vida de los pacientes con alteraciones vestibulares, las nuevas tecnologías, como la realidad virtual, parece que podría tener un papel importante en el tratamiento de estos pacientes. La realidad virtual parece reducir la sintomatología en pacientes con déficit cognitivo. Todo esto unido al actual abaratamiento de los costes, y por tanto a su mejor alcance, la realidad virtual puede ser una opción muy relevante para la rehabilitación vestibular.

El vértigo es un patología que consiste en una mala percepción de movimiento, el paciente nota que él o el espacio de su alrededor se está desplazando sin que en realidad exista movimiento.

Nuestro artículo está basado en un trabajo del Dr. Jae-Jun Song es un investigador, médico otorrinolaringólogo y profesor asociado a la Universidad de Korea. Sus principales investigaciones versan sobre la epidemiología del vértigo, así como su abordaje mediante nuevas tecnologías

Nuestra población está cada vez más envejecida y el mareo es un factor que se puede llegar asociar con la edad. En un estudio realizado por Murdin et al. (2015) se observó que la prevalencia del vértigo es de entre el 3 y el 10\%. Las intervenciones para el vértigo deben enfocarse de manera multifactorial y es necesario comprender varios factores para la prevención y el manejo de esta patología. La rehabilitación del aparato vestibular es un tratamiento que, a través del ejercicio, promueve: la compensación vestibular, es decir, el proceso de recuperación del aparato vestibular y proporciona la normalización del movimiento o situación que causa mareos, la mejora de la estabilidad, reduce el riesgo de caída, y puede llegar a mejorar la ansiedad asociada y los síntomas compatibles con la depresión.

La terapia de rehabilitación vestibular recientemente desarrollada proporciona tratamiento mediante la combinación de varios ejercicios, dependiendo de los síntomas y condiciones del paciente. La realidad virtual es un dispositivo capaz de transformar las señales producidas por una computadora en señales comprensibles para los humanos, que incluye simulación e interacción en tiempo real a través de varios y diferentes canales sensoriales, como la visión, el oído, el tacto, el olfato y el gusto. El grado de uso de la realidad virtual varía ampliamente, y estudios recientes han aplicado tecnologías de realidad virtual en el campo médico, incluyendo anatomía, modelos tridimensionales de pacientes, cirugía virtual, y rehabilitación.

Se han estudiado y desarrollado sistemas de rehabilitación que utilizan tecnologías de realidad virtual. Estos dispositivos en combinación con ordenadores portátiles pueden tener una amplia variedad de aplicaciones en el campo sanitario. Además, mediante la aplicación de diferentes contenidos tales como juegos, podemos llegar a motivar continuamente a los pacientes, descubriendo un tipo de rehabilitación mucho más agradable, atractivo, obteniendo un feedback constante por parte del paciente, llegando a observar una posible mejora en los resultados de la rehabilitación.

Se han desarrollado diferentes estudios sobre el uso de la realidad virtual para mejorar la eficiencia de la rehabilitación vestibular. Anteriormente, se utilizaban una unidad de rehabilitación del equilibrio, una plataforma de fuerza y una tabla de feedback denominada Wii Fit de la marca Nintendo, pero recientemente se ha

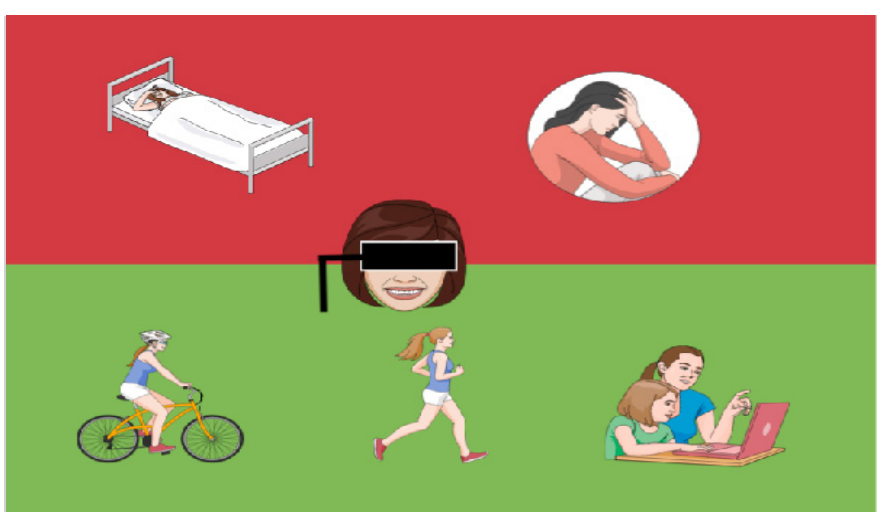

\section{"La realidad virtual aumenta la implicación en la rehabil- itación del paciente"}


desarrollado un equipo de inmersión que utiliza la pantalla frontal. Park y sus colaboradores han mostrado que, por ejemplo, los seguidores de movimiento y las placas de fuerza, que se denominan unidades híbridas de realidad virtual, son eficaces para la reducción de los síntomas que perciben los pacientes con disfunción vestibular periférica. También puede ser una opción muy segura de tratamiento para pacientes vestibulares con deterioro cognitivo, demostrando ser buen tratamiento para el reflejo vestíbulo-ocular (es un reflejo que produce un movimiento ocular en la dirección opuesta al movimiento de la cabeza, conservando la imagen en el centro del campo visual) y el control postural (capacidad de realizar cualquier acción, a pesar de la gravedad y evitar la caída) produciéndose así una mejora de la calidad de vida.

Otro estudio muestra que el dispositivo de realidad virtual en combinación con el seguimiento ocular y equipos de inmersión con pantalla frontal, puede aumentar el número de conexiones neuronales, a la vez que proporciona la rehabilitación vestibular.

Estos dispositivos de realidad virtual emplean diferentes juegos, aumentan la concentración, y resulta una buena opción para el tratamiento del paciente. Además, estimula múltiples sentidos simultáneamente y pueden aumentar la eficacia de la rehabilitación
"Debido al actual abaratamiento de sus costes, su fácil uso y su cantidad de diversas utilidades,

la realidad virtual es una gran opción para la rehabilitación vestibular"

y ayudar a tratar a los pacientes con problemas vestibulares. Los primeros modelos de dispositivos de realidad virtual no pudieron ser comercializados debido a que eran equipos costosos y de gran tamaño, pero recientemente, con la aparición de equipos de inmersión con cámara, se produce un abaratamiento del coste y una simplificación en su uso, pudiendo llegar a ser muy práctico y de gran ayuda en el futuro.

\section{Conclusión:}

Debido al envejecimiento de la población y su posible relación con los problemas vestibulares, la realidad virtual es una posibilidad de rehabilitación vestibular. Pudiendo ser utilizada por todo tipo de pacientes, implicándose más los afectados por esta patología en su recuperación, debido a las diferentes alternativas y juegos que nos aporta. La realidad virtual reduce la sintomatología en pacientes con déficit cognitivo, pudiendo incluso llegando a producir un aumento del número de conexiones neuronales. Todo esto unido al actual abaratamiento de los costes, su fácil manejo y su multitud de utilidades, la realidad virtual puede ser una opción muy competente para la rehabilitación vestibular.

\section{Sobre este artículo:}

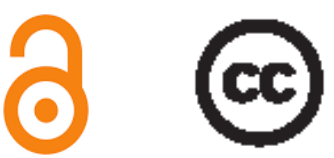

Fuente /s:

-Song, J. (2019). Virtual Reality for Vestibular Rehabilitation. Clinical And Experimental Otorhinolaryngology, 12;(4):329-330. doi: 10.21053/ceo.2019.00983

-Murdin, L., \& Schilder, A. G. M. (2015). Epidemiology of Balance Symptoms and Disorders in the Community. Otology \& Neurotology, 36;(3):387-392.

-Park JH, Jeon HJ, Lim EC, Koo JW, Lee HJ, Kim HJ, et al. Feasibility of eye tracking assisted vestibular rehabilitation strategy using immersive virtual reality. Clin Exp Otorhinolaryngol. 2019, 12;(4):376-84.

Fuente de la Imagen: imagen de NeuroRehabnews.com con fines unicamente ilustrativos.

Para citar este artículo: Carrasco-González E. Realidad virtual en el abordaje de alteraciones vestibulares. NeuroRehab News 2020 mar; 4(1):e0069

Enrique

Carrasco González

Edición: Ferran Cuenca Martínez y Roy La Touche 